\title{
Genetic Variations of Gliadin and HMW Glutenin Subunits in Spelt Wheat
}

\author{
Zhi-En Pu, Yu-Ming Wei, You-Liang Zheng* \\ Triticeae Research Institute, Sichuan Agricultural University Dujiangyan, 611830, China
}

Received: 15 December 2005. Accepted: 13 June 2006

\begin{abstract}
Gliadin and HMW glutenin subunit variations in 162 spelt wheat (Triticum spelta L.) accessions were detected by A-PAGE and SDS-PAGE. Higher gliadin variation was observed, and 121 gliadin patterns were detected. A total of 14 HMW-GS alleles were found. There were 2, 5 and 2 alleles at Glu-A1, Glu-B1 and Glu-D1 loci, respectively, resulting in 8 HMW-GS combinations. HMW-GS combination $(1,6+8,2+12)$ was the dominant phenotype, which was found in $83.00 \%$ of the spelt wheat accessions. These results suggested that the polymorphism of spelt wheat on Gli-1 loci was higher than that on Glu-1 loci.
\end{abstract}

Key-words: gliadin, HMW glutenin subunit, spelt wheat.

\section{Introduction}

Spelt wheat (Triticum spelta L.) is well recognized as one of the well-known wheat, which possesses the same genome (AABBDD) as bread wheat. The morphological character of spelt is well defined, typically characterized by a narrow, lax and pyramidal spike with a brittle rachis and adherent glumes, generally long spike internodes and non-spherical seeds (Winzeler et al., 1994). Spelt as a crop appears to quality for cultivation in less tolerant environmental condition like the high altitude regions as well as for low-input farming. Moreover, the ever-increasing demand for natural or organically grown foods creates a niche market for spelt and stimulates research into their utilization in traditional and new nutritious (Cubadda et al., 1996; Abdel-Aal et al., 1998), attractive foods such as breakfast cereals and extrude cooked products (Auricchio et al., 1982; Strehlow et al., 1991). In addition, spelt has other excellent characters like disease resistance, flooding tolerance (Burgos et al., 2001). The spelt wheat also is an important source of genetic diversity for endosperm proteins that are associated with bread-making quality in wheat. In a word, the interest in this crop is growing gradually.
Biochemical and genetic aspects of wheat storage proteins had received great attention due to their importance in determining the nutritional and technological properties of cultivated wheats. Gliadin and glutenin are the main storage proteins in wheat endosperm and are encoded by genes at Gli-1 and Glu-1 loci, respectively (Payne et al., 1982; Payne et al., 1983). Variations at each Glu-1 locus have been extensively characterized in the past two decades, mainly due to their relationships with breadmaking quality (Payne et al., 1987). On the other hand, storage protein polymorphisms are also useful genetic markers for crop origin (Shewry et al., 1992) and evolutionary studies (Fernandez-Calvin and Orellana, 1990). APAGE and SDS-PAGE have been used frequently to fractionate wheat gliadins and glutenin for identification of genotypes, establishment of chromosomal control, selection of desirable wheat lines in breeding programs, and/or the correlation of individual proteins with end-use quality.

The objective of this study was to detect the gliadin and glutenin variations in 162 spelt wheat accessions derived from various countries.

\footnotetext{
*Corresponding Author: Tel. e Fax: +86 1084242354. E-mail address: ylzheng@sicau.edu.cn
} 


\section{Materials and methods}

\subsection{Plant materials}

The gliadins and HMW-glutenin variations in 162 spelt wheat accessions, derived from various countries, were analyzed in this study (Table 1).

\subsection{Electrophoresis}

Gliadin proteins were extracted from single seeds with a solution of $70 \%(\mathrm{~V} / \mathrm{V})$ ethanol and $0.01 \%(\mathrm{~W} / \mathrm{V})$ methyl green, and fractionated by a standard acid polyacrlamide gel electrophoresis (A-PAGE) at pH 3.1 according to Wei et al. (2000).

HMW-glutenins were extracted from indi- vidual grains using the sequential procedure of $\mathrm{Ng}$ and Bushuk (1987). Electrophoresis of HMW-glutenins was performed on vertical gel according to the SDS-PAGE protocol described by $\mathrm{Ng}$ and Bushuk (1987). Common wheat variety Chinese Spring was used as a reference, and HMW-glutenin subunits were identified according to Payne and Lawrence (1987) and Ciaffi et al. (1991).

\section{Results and discussion}

\subsection{Gliadin variations}

One hundred and twenty-one gliadin patterns were detected in 162 spelt wheat accessions (Fig. 1). It was obvious that there were differ-

Table 1 . Origin of 162 spelta wheat accessions.

\begin{tabular}{|c|c|}
\hline Country & Accesions \\
\hline United States & $\begin{array}{l}\text { CItr1772, CItr1774, CItr2986, CItr3264, CItr13959, CItr13967, CItr15071, CItr17764, PI168682, } \\
\text { PI294576, PI355656, PI355595 }\end{array}$ \\
\hline Spain & PI190960, PI190962, PI190963, PI191100 \\
\hline Ethiopia & PI191392 \\
\hline Portugal & PI191617 \\
\hline Poland & PI192717, PI286060 \\
\hline Yugoslavia & PI221404, PI221419, PI221420 \\
\hline Iran & PI225271, PI225295 \\
\hline United Kingdom & PI266848, PI289607, PI330558, PI330559 \\
\hline Hungary & $\begin{array}{l}\text { PI272529, PI272573, PI272574, PI272576, PI27257, PI272578, PI272579, PI290513, PI290514, } \\
\text { PI290515, PI290516 }\end{array}$ \\
\hline Bulgaria & $\begin{array}{l}\text { PI295056, PI295059, PI295060, PI295061, PI295062, PI295063, PI295064, PI295066, PI295067, } \\
\text { PI295068, PI295069 }\end{array}$ \\
\hline Germany & $\begin{array}{l}\text { PI286048, PI355611, PI355612, PI355613, PI355614, PI355615, PI355618, PI355622, PI355623, } \\
\text { PI355624, PI355626, PI355633, PI355636, PI355639, PI355640, PI355641, PI355648, PI355649, } \\
\text { PI355657, PI355658, PI355660 }\end{array}$ \\
\hline Ethiopia & PI297861 \\
\hline Romania & PI306550, PI306551, PI306553, PI306554, PI306555, PI306556 \\
\hline Austria & PI323438 \\
\hline Belgium & $\begin{array}{l}\text { PI338366, PI338367, PI228368, PI355619, PI355620, PI355621, PI355625, PI355634, PI355635, } \\
\text { PI355650, PI355659 }\end{array}$ \\
\hline Argentina & PI346853 \\
\hline Switzerland & $\begin{array}{l}\text { PI347850, PI355591, PI355592, PI355593, PI355594, PI355596, PI355597, PI355598, PI355599, } \\
\text { PI355600, PI355601, PI355602, PI355603, PI355604, PI355605, PI355606, PI355607, PI355608, } \\
\text { PI355609, PI355610, PI355616, PI355617, PI355627, PI355628, PI355629, PI355630, PI355631, } \\
\text { PI355632, PI355637, PI355638, PI355643, PI355644, PI355645, PI355646, PI355647, PI355652, } \\
\text { PI355653, PI355654, PI355655, PI347851, PI347852, PI347853, PI347854, PI347855, PI34785, } \\
\text { PI347857, PI347858, PI347859, PI347860, PI347861, PI347862, NGB4495 }\end{array}$ \\
\hline Austria & PI355651 \\
\hline Denmark & $\begin{array}{l}\text { NGB4798, NGB5148, NGB5149, NGB9004, NGB9005, NGB9027, NGB9028, NGB9047, } \\
\text { NGB9055, NGB9056, NGB9680, NGB9700, NGB10883 }\end{array}$ \\
\hline Unknown & CItr14138, As327, As328 \\
\hline
\end{tabular}




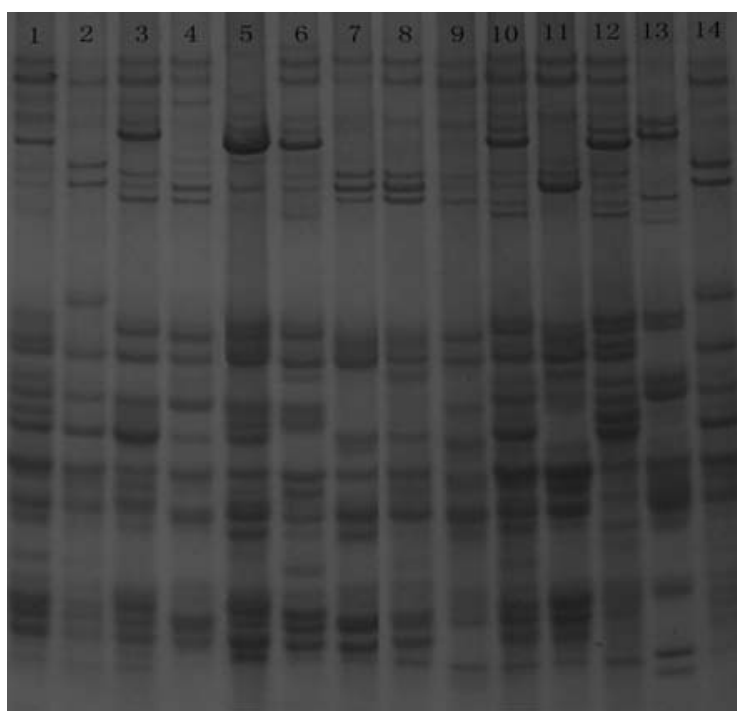

Figure 1. Gliadin patterns of the spelt wheat accessions CItr1774 (1), CItr2968 (2), CItr3264 (3), CItr14138 (4), CItr17764 (5), PI168682 (6), PI225271 (7), PI266848 (8), PI272529 (9), PI272573 (10), PI272574 (11), PI272576 (12), PI27257 (13) and PI27258 (14).

ences in number of $\omega / \gamma$-gliadin bands and their relative motilities among these spelt wheats. Seventeen gliadin bands were found in the $\omega$ gliadin zone, resulting in $48 \omega$-gliadin patterns. The number of $\omega$-gliadin bands varied from 4 to 8 in each accession. Diagrammatic of $\omega$ gliadin patterns and its frequency were shown in figure 2. The patterns 1 and 2 were the most frequent patterns, and observed in 20 and 15 accessions, respectively. A few patterns also had relatively higher frequency, such as 3, 4, 5 and 6 , whereas $20 \omega$-gliadin patterns were unique. A total of 10 gliadin bands were detected in the $\gamma$ gliadin zone, ranging from to 1 to 5 bands per accession (Fig. 3). There were $24 \gamma$-gliadin pat- terns, among which 10 patterns were unique. However, the relatively lower variations were detected in $\alpha$ - and $\beta$-gliadin zones. There were only 4 and 8 patterns in $\alpha$ - and $\beta$-gliadin zones, respectively (data not shown). Most of these spelt wheat accessions shared the identical $\alpha$ and $\beta$-gliadin bands. It was found that $\omega$ - and $\gamma$ gliadins had higher diversity than that of $\alpha$ - and $\beta$-gliadins (Abdel-Aal et al., 1996). In spelt wheat, it was reported that the gliadins encoding by $1 \mathrm{~A}, 1 \mathrm{~B}$ and $1 \mathrm{D}$ (i.e. $\omega$ - and $\gamma$-gliadins) had higher genetic variability than that of encoding by $6 \mathrm{~A}, 6 \mathrm{~B}$ and $6 \mathrm{D}$ (i.e. $\alpha$ - and $\beta$-gliadins) (Baker and Bushuk, 1978; Caballero et al., 2004b). In this study, the results were in agreement with that of Baker and Bushuk (1978) and Caballero et al. (2004b).

\subsection{HMW-glutenin variations}

A total of 14 HMW-GS alleles were found among the 162 spelt wheat accessions (Fig. 4). There were 2, 5 and 2 alleles at Glu-A1, Glu$B 1$ and Glu-D1 loci, respectively, resulting in 8 HMW-GS combinations (Table 2). It was obvious that HMW-GS combination $(1,6+8,2+12)$ was the dominant phenotype, which was found in $83.00 \%$ of the spelt wheat accessions. $\mathrm{Ni}$ (2002) reported that Glu-B1d (6+8) was the preponderant subunits of spelt wheat $(57.1 \%)$, whereas Caballero et al. $(2001,2004)$ and Rodriguez-Quijano et al. (1990) found that GluB1f $(13+16)$ was the dominant subunits $(87.8 \%)$. In this study, we observed that Glu$B 1 d(6+8)$ was the preponderant subunits.

In this study, the Glu-Alc allele was rare (frequency $2.5 \%$ ) and found in only 4 accessions (i.e. PI295056, PI355640, NGB9004 and NGB9027), whereas higher frequency of Glu-

Table 2. HMW-glutenin subunits combinations in 162 spelt accessions.

\begin{tabular}{lcclc}
\hline Glu-A1 & Glu-B1 & Glu-D1 & Accessions & Frequency (\%) \\
\hline 1 & $6+8$ & $5+10$ & PI355608 & 0.62 \\
1 & 20 & $2+12$ & PI355594 & 0.62 \\
1 & $6^{*}+8^{*}$ & $2+12$ & PI355612 & 0.62 \\
1 & $6.1+22.1$ & $2+12$ & PI347850 & 0.62 \\
null & $6+8$ & $2+12$ & PI355640; NGB9004 & 1.24 \\
null & $7+8$ & $2+12$ & NGB9027; PI295056 & 1.24 \\
1 & $7+8$ & $2+12$ & NGB9700; PI355615; As328; NGB5148; As327; PI295060; & \\
& & & PI272576; PI190960; PI355621; PI355622; PI221420; PI355605; & \\
& & & NGB9680; PI355597; PI191717; PI355623; PI272579; NGB9028; & 12.04 \\
1 & $6+8$ & $2+12$ & The remaining accessions & 82.72 \\
\hline
\end{tabular}




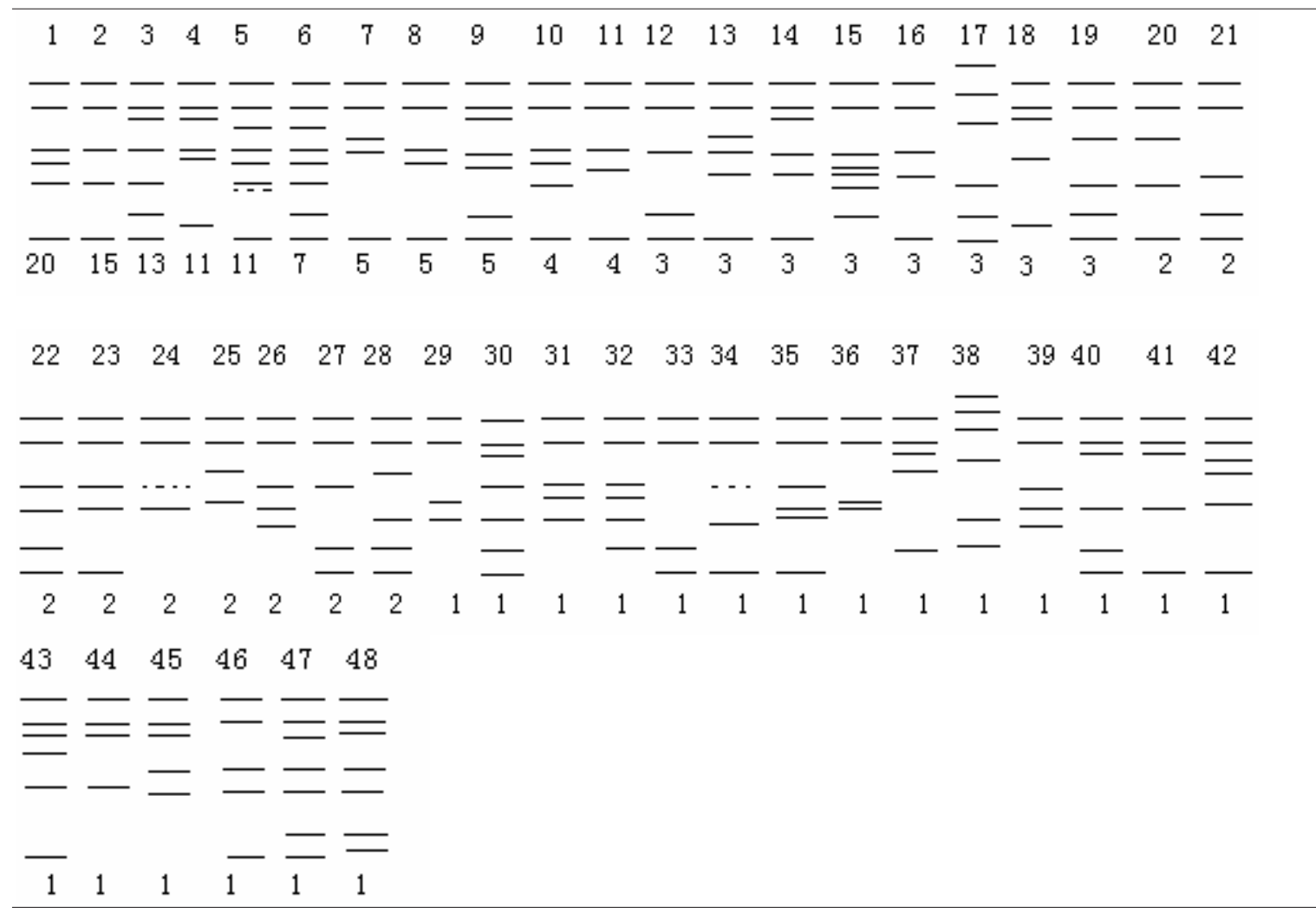

Figure 2. Diagrammatic representation of the patterns in the $\omega$-gliadins zone. The bands with light trace are shown with discontinuous line. The patterns are numbered from 1 to 48 , in decreasing order to the presence among all accessions, and the number under the patterns indicated the number of accessions that the patterns present.

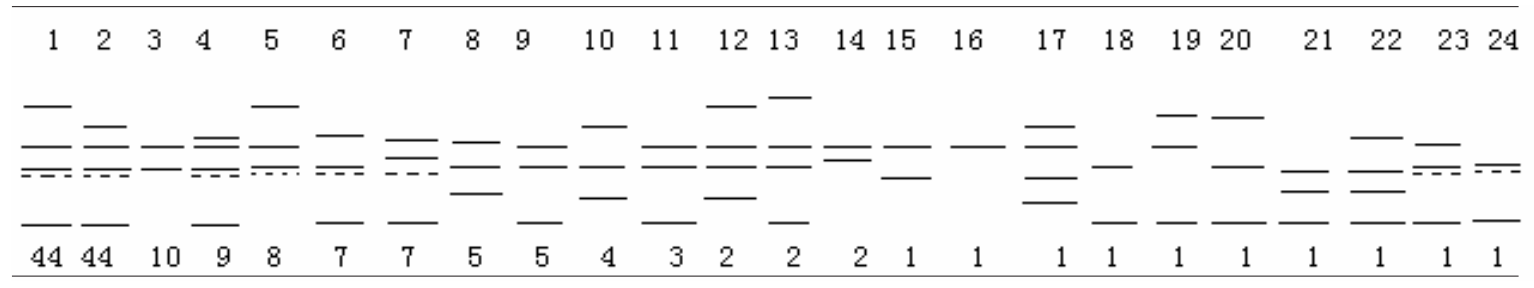

Figure 3. Diagrammatic representation of 24 patterns of $\gamma$-gliadins zone. The patterns are numbered similar to figure 2.

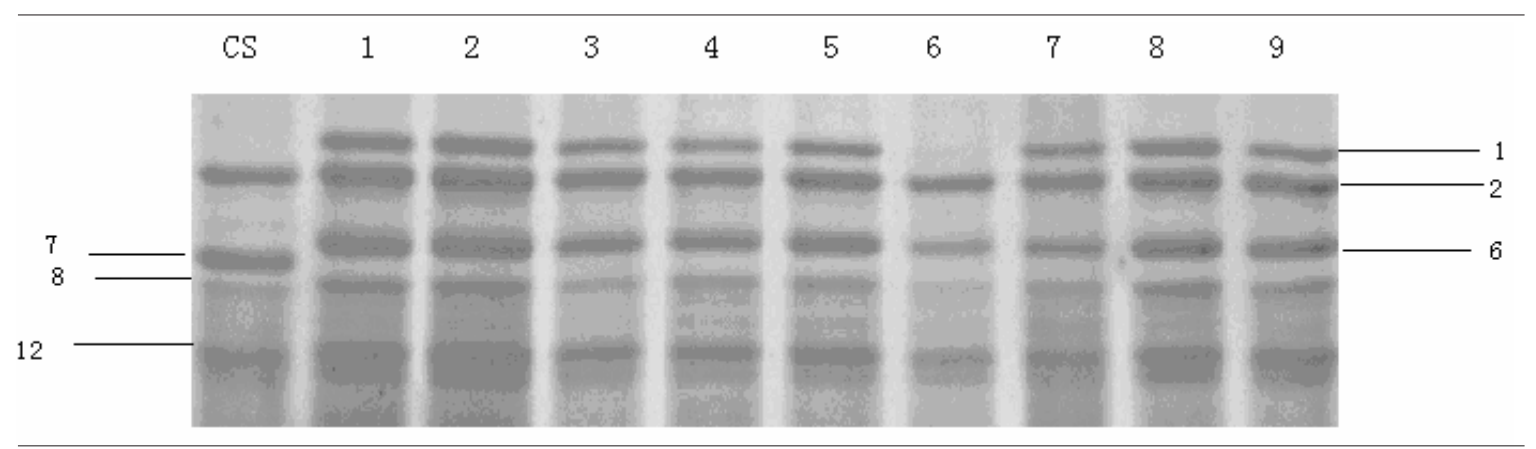

Figure 4. HMW-glutenin subunits of spelt wheat accessions PI355635 (lane 2), PI355636 (lane 3), PI355637 (lane 4), PI355638 (lane 5), PI355639 (lane 6), PI355640 (lane 7), PI355641 (lane 8), PI355642 (lane 9) and PI355643 (lane 10). Chinese Spring (lane 1) (subunits: null, $7+8,2+12$ ) was used as a reference. 
A1c allele was found by Rodriguez-Quijano et al (1990). The subunit 2* coded by the Glu-A1b allele, which appeared with higher frequency in the findings of Rodriguez-Quijano et al. (1990) and Caballero et al (2001, 2004), were not found in this study. In addition, we found that the allele of Glu-A1 in PI190963 was subunit 1 other than "null" as reported by Caballero et al. (2001).

The allele GluB1b (subunits 7+8) associated with good bread-making quality (Payne and Lawrence1983), were rare in these analyzed spelt wheat accessions. The subunit 20 (GluB1e) was only found in one accession (PI355594), being classified as rare with a local distribution, and all these alleles had been observed in drum and bread wheat.

In 162 spelt wheat accessions, a higher homogeneity for the Glu-D1 locus was observed, and $99.4 \%$ of spelt wheat accessions had the Glu-D1a allele (subunits 2+12). The subunits $5+10$ (Glu-D1d), associated with good quality, was only detected in one accession PI355614.

\section{Acknowledgements}

This work was supported by the Hi-Tech Research and Development (863) Program of China (2003AA207100) and the Foundation for the Author of National Excellent Doctoral Dissertation of China (200357). Y.-L. Zheng was supported by Program for Changjiang Scholars and Innovative Research Team in University (IRT0453), China.

\section{References}

Abdel-Aal E.S.M., Hule P., Sosulski F.W., Bhirud P.R. 1997. Kernel, milling and baking properties of springtype spelt and einkorn wheats. Journal Cereal Science, 26:363-370.

Abdel-Aal E.S.M., Salama D.A., Hule P., Sosulski F.W., Cao W. 1996. Electrophoretic characterization of spring spelt wheat gliadins. Journal Agricultural Food Chemistry, 44:2117-2123.

Auricchio S., De Ritis G., De Vincenzi M., Occorsio P., Silano V. 1982. Effects of gliadin derived peptides from bread and durum wheats on small intestine cultures from rat fetus and the necessity of the protection and conservation of coelic children. Pediatr. Res., 16:1004.

Baker R.J., Bushuk W. 1978. Inheritance of differences in gliadin electropherograms in the progeny of 'Neepawa' and 'Pitic 62' wheats. Canada Journal Plant Science, 58:325-329.

Burgos M.St., Messmer M.M., Stamp P., Schmid J.E.
2001. Flooding tolerance of spelt (Triticum spelta L.) compared to wheat (Triticum aestivum L.) - A physiological and genetic approach. Euphytica, 122:287295.

Caballero L., Martin L.M., Alvarez J.B. 2001. Allelic variation of the HMW glutenin subunits in Spanish accessions of spelt wheat (Triticum aestivum ssp. spelta L. em. Thell). Theoretical Applied Genetics, 103:124128.

Caballero L., Martin L.M, Alvarez J.B. 2004a. Variation and genetic diversity for gliadins in Spanish spelt wheat accessions. Genetic Resources Crop Evolution, 51:679-686.

Caballero L., Martin L.M, Alvarez J.B. 2004b. Intra- and interpopulation diversity for HMW glutenin subunits in Spanish spelt wheat. Genetic Resources Crop Evolution, 51:175-181.

Ciaffi M., Lafiandra D., Porceddu E., Benedettelli S. 1993. Storage protein variation in wild wheat (Triticum turgidum ssp. dicoccoides) from Jordan and Turkey. I. Electrophoretic characterization of genotypes. Theoretical Applied Geneticss, 86:474-480.

Cubadda R. 1996. Pasta quality: The relationship between raw material properties and production technologies. In: Proc. $1^{\text {st }}$ World Pasta Congress, 164-168. Chiriotti Editori, Pinerolo, Italy.

Dvork I., Luo M.-C., Yang Z.-L., Zhang H.-B. 1998. The structure of the Aegilops tauschii genepool and the evolution of haxapolid wheat. Theoretical Applied Genetics, 97:127-136.

Fernandez-Calvin B., Orellana J. 1990. High-molecularweight glutenin subunit variation in the Sitopsis section of Aegilops. Implications for the origin of the B genome of wheat. Heredity, 65:455-463.

Ng P.K.W, Bushuk W. 1987. Glutenin of Marquis wheat as a reference for estimating molecular weights of glutenin subunits by sodium dodecyl sulfate-polyacrylamide gel electrophoresis. Cereal Chemistry, 64:324-327.

Ni Z., Sun Q., Zhang Y., Liu G. 2002. High molecular weight glutenin subunits composition of spelt wheat. Journal Agriculture Biotechnology, 10:108-112.

Payne P.I., Holt L.M., Lawrence G.J., Law C.N. 1982. The genetics of gliadin and glutenin, the major storage proteins of the wheat endosperm. Qualitas Plantarum Plant Foods Human Nutrition, 31:229-241.

Payne P.I., Lawrence G.J. 1983. Catalogue of alleles for the complex loci, Glu-A1, Glu-B1 and Glu-D1 which coded for high-molecular-weight subunits of glutenin in hexaploid wheat. Cereal Research Communications, 11:29-35.

Payne P.I. 1987. The genetical of basis of bread-making quality in wheat. Aspects Applied Biology, 15:79-90.

Sun Q., Wei Y., Ni Z., Xie C., Yang T. 2002. Microsatellite marker for yellow rust resistance gene Yr5 in wheat introgressed from spelt wheat. Plant Breeding, 121:539-541. 
Rodriguez-Quijano M., Vázquez J.F., Carrillo J.M. 1990. Variation of high-molecular-weight glutenin subunits in Spanish landraces of Triticum aestivum ssp. vulgare and ssp. spelta. Journal Genetics Breeding, 44:121-126.

Shewry P.R., Halford N.G., Tatham A.S. 1992. High-molecular-weight subunits of wheat glutenin. Journal Cereal Science, 15:105-120.

Strehlow W., Hertzka G., Weuffen W. 1991. The dietary in properties of spelt in the treatment of chronical diseases. In: Proc. 2. Hohenheimer Dinkel Kolloqui- um, Hohenheim, 243-259. Alemania Verlag, Stuttgart.

Winzele H., Schmid J.E., Winzeler M. 1994. Analysis of the yield potential and yield components of F1 and F2 hybrids of crosses between wheat (Triticum aestivum L.) and spelt (Triticum spelta L.). Euphytica, 74:211-218.

Yu-ming Wei, You-liang Zheng, Deng-Cai Liu, YongHong Zhou, Xiu-Jin Lan 2000. Gliadin and HMWglutenin variation in Triticum turgidum L. ssp. turgidum and $T$. aestivum $\mathrm{L}$. landraces native in Sichuan, China. Wheat Information Service, 90:13-20. 